Studientext für Studierende in Fächern mit einem Medienbezug. (Einen solchen Band habe vermutlich nicht nur ich bislang für meine Lehraufgaben vermisst.)

Nachdem im vorliegenden Buch solche Unterhaltungsangebote eine Würdigung fanden, die auch im Sinne von Dehm (1984) zweifellos von den Rezipienten als Unterhaltungssendungen eingeschätzt werden würden, wäre ich an einem weiteren Band interessiert, der den Unterhaltungsaspekt solcher Medienangebote zum Gegenstand hat, die vordergründig der Information gewidmet sind (z. B. Quizsendungen, Lernsoftware, Lehrfilme, Nachrichten). Einen solchen Sammelband (vom gleichen Herausgeberteam?) könnte ich mir sehr gut als Fortsetzung des hier besprochenen Werkes vorstellen.

Roland Mangold

Literatur:

Berghaus, Margot (1994). Wohlgefallen am Fernsehen. Eine Theorie des Gefallens in Anlehnung an Immanuel Kant. Publizistik, $39,141-160$.

Dehm, Ursula (1984). Fernsehunterbaltung. Zeitvertreib, Flucht oder Zwang? Mainz.

Früh, Werner, Kuhlmann, Christoph $\&$ Wirth, Werner (1996). Unterhaltsame Information oder informative Unterhaltung? Publizistik, 41, $428-451$.

Klaus, Elisabeth (1996). Der Gegensatz von Information ist Desinformation, der Gegensatz von Unterhaltung ist Langeweile. Rundfunk und Fernsehen, 44, $402-417$.

Mangold, Roland (2000). Der abendliche Horror? Unterhaltung und Emotionen bei Fernsehnachrichten. In: Gunnar Roters, Walter Klingler \& Maria Gerhards (Hrsg.), Unterhaltung und Unterhaltungsrezeption (S. 119 - 140). Baden-Baden.

Winterhoff-Spurk, Peter (2000). Der Ekel vor dem Leichten. Unterhaltungsrezeption aus medienpsychologischer Perspektive. In Gunnar Roters, Walter Klingler \& Maria Gerhards (Hrsg.), Unterbaltung und Unterhaltungsrezeption (S. 77 - 98). BadenBaden.

\section{Eggo Müller}

\section{Paarungsspiele}

Beziehungsshows in der Wirklichkeit des neuen Fernsehens

Berlin: Edition Sigma, 1999. - 262 S.

(Sigma Medienwissenschaft; 25)

Zugl.: Hildesheim, Univ., Diss., 1998

ISBN 3-89404-919-7

Die Fernsehshow lebt. Das Jahr 2000 hat uns einen neuen Boom beschert: Quizshows feiern ein Come Back. Insbesondere die EndemolProduktion „Wer wird Millionär" veranlasst zur Zeit ein Millionenpublikum zum Mitspielen und Mitfühlen, unabhängig von Alter, Geschlecht oder Bildung. Und in fast jeder geselligen Runde findet man Menschen, die sich schon als Kandidat oder Kandidatin beworben haben. Das Quizshow-Revival folgt auf den Boom der Beziehungsshows, die die Fernsehunterhaltung der 1990er Jahre prägten. Spiele um Wissen, Geld und gute Nerven sind damit neben die Spiele um Beziehungen und Emotionen getreten - Feuilletonredakteure nehmen es wohlwollend bis erleichtert zur Kenntnis. Jedoch ist die Beziehungsshow keineswegs tot. Zum einen haben unbesehen einer gewissen Marktbereinigung diverse Shows überlebt. So auch die Singleshow „Herzblatt“ (ARD), von der ausgehend Eggo Müller seine Analyse über „Beziehungsshows in der Wirklichkeit des neuen Fernsehens" entfaltet. Zum anderen wurden einige ihrer Kennzeichen in Containershows wie ,Big Brother' überführt und radikalisiert - insbesondere „die spezifische Vermischung sozialer und medialer Wirklichkeiten“ (19).

Müller nähert sich dem Phänomen Beziehungsshow am Beispiel ,Herzblatt' von zwei Seiten: Zum einen analysiert er die spezifische Form und Ästhetik des Genres mittels einer „Strukturanalyse, die von der Rahmenkonstruktion und dem daraus folgenden kommunikativen Potenzial von Beziehungsshows ausgeht" (S. 12). Zum anderen betrachtet der Autor die gesellschaftlichen Kontexte, in denen diese Shows agieren und Resonanz erzeugen: „So wenig die ,großen' soziologischen Fragen jenseits der spezifischen Formen der Shows erörtert werden können, so wenig können diese Formen jenseits ihres sozialen Rahmens verstanden werden." (11) Diese Verbindung von ästhetischer und sozialer Perspektive macht 
den Reiz der Studie aus und vermittelt Erkenntnisgewinne auch über das behandelte Genre hinaus.

Methodisch ordnet Müller seine Studie als pragmatisch orientierte Analyse im Rahmen der Cultural Studies ein, die nicht auf die Bedeutungen einzelner Sendungen, sondern auf die Strukturen des Genres als Bedingungsrahmen für Sinnbildungsprozesse zielt. (Kapitel 1). Im Rückgriff auf Goffmans Rahmenanalyse widmet sich Müller zunächst den Arrangements von „Herzblatt“ als „Prototyp“ des Genres (Kapitel 2). In dieser Singleshow wählt jeweils ein Kandidat eine Frau oder eine Kandidatin einen Mann für eine Wochenendreise zu zweit aus. Dazu werden den zur Auswahl stehenden Menschen drei Fragen gestellt, sie können aber nicht in Augenschein genommen werden, weil sie zunächst hinter einer Wand sitzen. Das Publikum verfügt über mehr Informationen als die Kandidatin oder der Kandidat. Auf der Ebene des Spiels eröffnet sich damit eine spezifische Rezeptionsmöglichkeit: Das Publikum wird hier nicht etwa zum Mitspieler wie bei Quizshows, denn es hat gegenüber den Kandidaten einen Wissensvorsprung. Es kann vielmehr Prognosen stellen, wer ausgewählt wird, ob das Paar zueinander passen wird u. a. m. - einen vergleichbaren Zugang bietet übrigens auch ,Big Brother', und dabei spielt in beiden Formaten die Auswahl und typisierende Inszenierung der Mitspielenden durch die Redaktion eine wichtige Rolle. Müller findet neben dieser Spielebene zwei weitere dramatische Ebenen der Show: Romanze und Comedy. Romanze bezieht sich auf die mögliche Anbahnung einer Beziehung zwischen den ShowKandidatInnen und somit auf die Ebene sozialer Folgenhaftigkeit im ,wirklichen' Leben. Als Comedy analysiert der Autor die vorfabrizierten, mit Stereotypen und sexuellen Anspielungen versehenen Fragen und Antworten der Mitspielenden. Changierende Rahmen prägen auch die Kandidatenrolle: In der „Performance" der KandidatInnen überlagern sich drei Modi der Darstellung, indem "diese auf der Show-Bühne jederzeit gleichzeitig sie selbst sind, sich selbst darstellen und die Rolle des Kandidaten geben“ (89). Gemischte Wirklichkeiten und eine mehrschichtige Performance geben der Show demnach ihren zugleich künstlichen wie wirklichen Charakter. Dieser Befund gilt auch für das Genre der Beziehungsshows insgesamt, deren Spielarten Müller unter der Überschrift „Beziehungs-Arrangements in gemischten Wirklichkeiten" analysiert (Kapitel 3). Als „Interaktionsshows“ weisen sie zwei zentrale Momente auf: zum einen die zugrundeliegende soziale Situation, zum anderen das mediale Arrangement, das die Interaktionen unter der Bedingung möglicher sozialer Folgen organisiert (107).

Warum wurde das Genre zum Gegenstand der Unterhaltung im Fernsehen der 90er Jahre? Antworten findet der Autor in der Analyse der gesellschaftlichen Rahmen, die das Genre - mit Stuart Hall gesprochen - ,artikuliert“ (Kapitel 4). Das Genre Beziehungsshow versteht Müller ebenso „als Effekt wie als Moment des sozialen Wandels“ (145). Ökonomisch steht der Erfolg der Shows in einer engen Beziehung zum kommerzialisierten Fernsehen der 1990er Jahre: Sie sind relativ günstig zu produzieren und sprechen vor allem das für die Werbewirtschaft attraktive Zielpublikum der konsumfreudigen 14- bis 29-Jährigen an. Gesellschaftlich werden die Shows, anknüpfend an die Analysen von Beck und Beck-Gernsheim sowie Giddens, im Rahmen von Prozessen der Modernisierung und Individualisierung verortet. Der Single symbolisiert diese Entwicklungen idealtypisch. Beziehungsshows artikulieren die Auseinandersetzung um die Bestimmung einer individuellen Identität. Die (symbolische) Gestaltung einer heterosexuellen Paar-Beziehung bildet den Stoff der Beziehungsshows, Geschlechterbeziehung und Sexualität stellen ihren zentralen gesellschaftlichen Rahmen dar. Müller versteht die Shows als Rituale zur (Re)Inszenierung und Stabilisierung der binären Ordnung der Geschlechter. Das heterosexuelle Paar wird „in den Aufführungen der Beziehungsshows als eine quasi natürliche soziale Einheit manifestiert und seine Geltung als stilles Hintergrundwissen des Alltags gesichert“ (180). Durch ihre „Hyperritualisierung“ (176) werden die geltenden Darstellungskonventionen der Geschlechter einschließlich ihrer Veränderungen und Variationen offenbar gemacht. Dabei orientieren sich die Shows symbolisch an der Vorstellung einer gleichberechtigten, von Zwängen und Verantwortlichkeiten befreiten „reinen Beziehung“ (Giddens) und einer selbst genügsamen, hedonistischen Sexualität. Damit sind die Beziehungsshows keineswegs gesellschaftliche Vorreiter. Wie Müller zutreffend feststellt, können solche Tendenzen am Ende des 20. Jahrhunderts nicht als subver- 
siv gelten. Der Autor widerspricht in diesem Zusammenhang dem Subversivitätsverständnis einiger Cultural Studies-Ansätze und verortet die Shows im Einklang mit gesellschaftlichen Prozessen. Abschließend stehen Begriff und Grenzen der Unterhaltung zur Diskussion (Kapitel 5). Das Genre fungiert demnach als „Schaubühne der Lebensführung“ (204), zugleich darf jedoch die Grenze zum „sozialen Ernst“ (209) nicht (zu weit) überschritten werden.

Das Buch bietet eine anregende Lektüre, die durch ausgewiesene anstelle sporadisch versteckter Zusammenfassungen noch erleichtert worden wäre. Methodisch erweitert die Studie die oft zu engen Perspektiven kommunikationswissenschaftlicher Inhaltsanalysen, in denen mediale Arrangements und symbolische Bedeutungen häufig vernachlässigt werden. Die Genreanalyse erschließt ein Stück Fernsehgeschichte. Einige der herausgearbeiteten Charakteristika der Beziehungsshows führen direkt zu den seit 2000 populären Containerformaten. Auch hier bezeichnet, Reality' nicht die, wirkliche' Wirklichkeit, sondern ein „mediales Phänomen von ganz eigener Wirklichkeit“, das mit der außermedialen spielt (93).

Jutta Röser

\section{Klaus Merten}

\section{Einführung in die Kommunikationswissen- schaft}

Bd. 1: Grundlagen der Kommunikationswissenschaft

Münster: Lit, 1999. - 585 S.

(Aktuelle Medien- und Kommunikationsforschung ; 1)

\section{ISBN 3-89473-592-9}

Wird ein Buch als Einführung tituliert, dann verbindet man damit gewisse Erwartungen. Eine Einführung richtet sich an Studenten, vor allem an solche in den Anfangssemestern. Für diese Gruppe sollte sie einen Nutzen bringen. Eine Einführung muss sich also in ihrer Darstellung und in der Auswahl der Themengebiete an den Bedürfnissen von Studierenden im Grundstudium orientieren. Einführungen sollten einigermaßen umfassend und aktuell sein, die wichtigsten Themengebiete, Fragestellungen und Erkenntnisbestände des Faches sollten vermittelt werden. Den Qualitätskriterien Klarheit und Verständlichkeit kommt eine gesteigerte Bedeutung zu. Einführungen sollten den Stand der Wissenschaft klar, nachvollziehbar und möglichst neutral darstellen. Die vorliegende von Klaus Merten verfasste „Einführung in die Kommunikationswissenschaft" hat vor diesem Hintergrund einige Verdienste, leider bleibt sie aber auch einiges schuldig. Auch wenn es viele gelungene Passagen in der Arbeit gibt, so wiegen die Schwächen doch sehr schwer.

Besonders auffällig ist die mangelnde Aktualität der Arbeit. Im Literaturverzeichnis finden die 90 er Jahre kaum statt. Es scheint, als hätte es im letzten Jahrzehnt keinen wissenschaftlichen Fortschritt im Fach gegeben. Dann wäre es wohl aber nicht die rechte Zeit, um Einführungen zu schreiben, sondern eher die Zeit für Nekrologe. Nimmt man das Quellenverzeichnis zum Maßstab, dann gab es in den 90er Jahren nur relevante Forschungen zum Thema Öffentlichkeitsarbeit und zum Konstruktivismus Münsteraner Prägung. Diese Altertümlichkeit verwundert umso mehr, als der Autor selber schreibt, dass sich die Kommunikationswissenschaft im Wandel befinde. Im Wandel von der klassischen zur transklassischen Wissenschaft. Umso wichtiger wäre eine einigermaßen aktuelle Darstellung des Forschungsstandes. Sollte dieser Wandel etwa nur die oben dargestellten Felder berühren und ansonsten an der Kommunikationswissenschaft spurlos vorübergegangen sein? Die mangelnde Aktualität der Arbeit wird auch immer wieder in Details deutlich. Da wird auf Seite 185 die Entwicklung der Zahl der Medien eines großen Wirtschaftsunternehmens „von 1955 bis heute“ dargestellt. Heute bedeutet hier 1988. Ähnliches findet sich auch an anderen Stellen (vgl. etwa S. 268).

Den zweiten zentralen Kritikpunkt macht wohl eher unfreiwillig der Buchrücken deutlich. Danach handelt es sich um eine „Münsteraner Einführung“. Dies hat der Autor doch etwas $\mathrm{zu}$ wörtlich genommen. Hier werden nicht die Grundlagen der Kommunikationswissenschaft dargestellt, sondern vor allem die Münsteraner Fassung derselben. So intellektuell anregend und wissenschaftlich verdienstvoll der Konstruktivismus Münsteraner Prägung ist, so wenig kann es Sinn machen, in einem Lehrbuch, das doch wohl über die Münsteraner Grenzen hinaus Geltung beansprucht, einseitig diesen Standpunkt darzustellen. 